\title{
Identification of VIM and IMP genes and metallo-beta- lactamase enzymes in Escherichia coli isolates by molecular and phenotypic methods in shahrekord educational hospitals
}

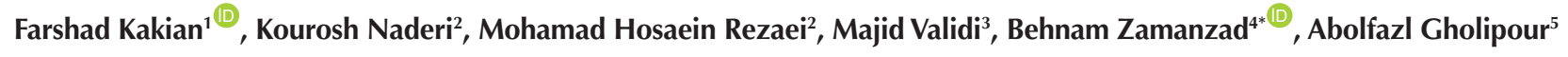 \\ 'Department of Bacteriology and Virology, School of Medicine, Shiraz University of Medical Sciences, Shiraz, Iran. \\ ${ }^{2}$ Cellular and Molecular Research Center, Shahrekord University of Medical Sciences, Shahrekord, Iran. \\ ${ }^{3}$ Department of Laboratory Sciences, School of Allied Medical Sciences, Shahrekord University of Medical Sciences, \\ Shahrekord, Iran. \\ ${ }^{4}$ Professor of Bacteriology, School of Medicine, Shahrekord University of Medical Sciences, Shahrekord, Iran. \\ ${ }^{5}$ Associate Professor of Bacteriology, Department of Microbiology and Immunology, School of Medicine, Shahrekord University \\ of Medical Sciences, Shahrekord, Iran.
}

*Corresponding Author: Behnam Zamanzad, Department of Microbiology and Immunology, Cellular and Molecular Research Center, Shahrekord University of Medical Sciences, Shahrekord, IR Iran, Tel: +983833346732, Fax: +98383334911, E-mail: bzamanzad@yahoo.com

\begin{abstract}
Background and aims: Among urine pathogens, Escherichia coli (E. coli) causes $80 \%$ of urinary tract infections (UTIs). Due to the destructive nature of penicillins, cephalosporins and carbapenems (except for monobactam such as aztreonam) and carbapenemase enzymes have created many problems for treating infectious diseases. Therefore, this study aimed to investigate the phenotypic and molecular characterization of metallo-beta-lactamase (MBL) genes produced by E. coli isolates in an educational hospital during 2016-2017.

Methods: This cross-sectional study investigated 80 UTI samples affected by $E$. coli. In addition, antibiotic susceptibility was evaluated by disk diffusion and E-test methods for two antibiotics of meropenem and imipenem. Phenotypic tests containing modified Hodge test, ethylenediaminetetraacetic acid (EDTA) disk synergy test, and AmpC Disk were performed to identify MBL enzyme-producing strains. Finally, the frequency of Verona integron-encoded metallo- $\beta$-lactamase (VIM) and imipenemase (IMP) genes was determined by polymerase chain reaction (PCR).

Results: Among $80 \mathrm{E}$. coli samples, $21(26.25 \%)$ isolates were resistant to meropenem and imipenem as detected by the disk-diffusion method and E-test. Further, phenotypic tests including modified Hodge test, EDS test, and AmpC disk test showed the positivity of 15 $(18.75 \%), 15(18.75 \%)$, and $8(10 \%)$ isolates, respectively $(P<0.001)$. Eventually, polymerase chain reaction (PCR) test results for the VIM gene showed $19(23.75 \%)$ positive isolates of $E$. coli, but the IMP gene was observed in none of the isolates $(P<0.001)$.

Conclusion: In general, the emergence of $E$. coli producing MBL enzymes is a serious threat among clinical infections. The findings of this study indicated the presence of $E$. coli producing MBL. These enzymes can degrade carbapenems antibiotics, the last class current treatment of multiple drug-resistance infections.

Keywords: Escherichia coli, Drug resistance, VIM, IMP
\end{abstract}

Received: 8 May 2019, Accepted: 20 June 2019, ePublished: 28 February 2020

\section{Introduction}

The family of Enterobacteriaceae, especially Escherichia coli, causes urinary tract infections (UTIs) (1). UTI is one of the most important factors in renal scarring, kidney tissue damage, kidney failure, poor growth, kidney stones, and hypertension (2-4). Almost 30\%-40\% of UTI are nosocomial infections which are considered as the most significant cause of sepsis in hospitalized patients (5). E. coli in the Enterobacteriaceae family causes hospital and acquired infections more than the other members of its family (6). In addition, E. coli is the most common etiologic agent of the UTI accounting for $80 \%$ of cases (7). Resistance to antibiotic therapy in patients with UTIs represents an example of the increasing problem of antimicrobial resistance (8). This type of resistance has always been a major problem for human health (9) and affects patients in general hospitals worldwide (10). Carbapenem antibiotics family consists of ertapenem, imipenem, doripenem, and meropenem. Nowadays, because of the broad spectrum and non-hydrolysis by beta-lactamases enzymes, this family has become the most important selected drug for treating serious infections in

(C) 2020 The Author(s); Published by Shahrekord University of Medical Sciences. This is an open-access article distributed under the terms of the Creative Commons Attribution License (http://creativecommons.org/licenses/by/4.0), which permits unrestricted use, distribution, and reproduction in any medium, provided the original work is properly cited. 
Enterobacteriaceae resistance to multiple drugs that produce extended broad-spectrum $\beta$-lactamases (ESBLs) (11-13). Carbapenemase is an important mechanism of resistance to carbapenem antibiotics. Further, resistance by metallobeta-lactamases (MBLs) often relies on the plasmid $(14,15)$. According to ambler classification, carbapenemase belongs to molecular classes A, B, and D. Class A carbapenemase includes Guiana-extended-spectrum, imipenemhydrolysing $\beta$-lactamase, Serratia marcescens enzyme, and Klebsiella pneumonia carbapenemase (KPC) enzymes that can be inhibited by varying degrees of clavulanate and they hydrolyse penicillins and cephalosporins more than carbapenems. Some of them, including KPC, lack the strong carbapenemase ability, which might be considered as ESBLs. Furthermore, carbapenemase class B is an MBL that is considered as group B of amblers based on the similarity of their amino acid sequences. Moreover, it can be classified into group 3 (Bush's classification) based on the structure of the substrate and the inhibitor component. MBL activity depends on zinc metal, which is capable of hydrolyzing beta-lactam antibiotics and all carbapenems except for aztreonam. MBLs are resistant to beta-lactamase inhibitors but can be prevented by chelating agents such as ethylenediaminetetraacetic acid (EDTA). According to molecular structures, MBLs are divided into 6 categories of Verona integron-encoded metallo- $\beta$-lactamase (VIM), imipenemase (IMP), German imipenemase, Seoul imipenemase (SIM), São Paulo metallo-beta-lactamase, and Adelaide imipenemase. The VIM, IMP, and SMP-1 were identified in Pseudomonas aeruginosa, Acinetobacter baumannii, and other members of the Enterobacteriaceae family (16-20). Considering the above-mentioned explanations, the aim of this study was to determine the frequency of VIM and IMP genes and MBL enzymes in E. coli isolates by molecular and phenotypic methods in educational hospitals of Shahrekord, Iran.

\section{Materials and Methods}

\section{Bacterial Isolates}

In this study, $80 \mathrm{E}$. coli isolates of UTI patients were prepared in several sections of the university hospital (e.g., urology, women, internal, intensive care unit) from May to October 2016. These patients were infected by UTI within 48-72 hours after hospitalization and the primary urine cultures were negative for them.

Antimicrobial susceptibility testing and minimum inhibitory concentration (MIC)

First, the samples were cultured on blood agar and eosinmethylene-blue mediums (Himedia) and then different biochemical identifier tests such as Triple sugar iron agar, Simon citrate, methyl red/Voges-Proskauer, SIM, and oxidase were performed on the isolates. In addition, antibiotic susceptibility was determined by the disk diffusion method (Kerby-Bauer) for meropenem and imipenem (Mast) and the plates were incubated for 18-24 hours at $37^{\circ} \mathrm{C}$. Finally, the Epsilon test (E-test) was used (Liofilchem Company) for two antibiotics of meropenem and imipenem on the Mueller-Hinton agar culture medium in order to determine the MIC.

\section{Phenotypic diagnosis of MBLs}

Phenotypic tests such as the modified Hodge test (MHT), EDTA disk synergy test (EDS), and AmpC disk test were performed to detect the strains containing MBL enzymes. Further, E. coli ATCC 25922 and Klebsiella pneumonia ATCC 1705 were considered as negative and positive controls for running MHT, respectively. Furthermore, bacterial suspension equivalent to $0.5 \mathrm{McFarland}$ was prepared from the fresh cultures of E. coli ATCC 25922 and then diluted in a 1:10 ratio and passaged on Mueller-Hinton agar. The plates were maintained at $37^{\circ} \mathrm{C}$ overnight. The appeared clover leaf shape in the inhibition site of bacterial growth resulted from the production of carbapenemase by the tested bacteria strain (21-23). Moreover, 0.5 molar EDTA solution and meropenem disk were used in the EDS test, followed by passaging sample suspensions equivalent to $0.5 \mathrm{McF}$ arland on Mueller-Hinton agar. A meropenem $(10 \mu \mathrm{g})$ disk was placed on the plate and then $10 \mathrm{~mL}$ of 0.5 molar EDTA solution was added to a blank disk (filter paper with a diameter of $6 \mathrm{~mm}$ ) in an aseptic condition. Additionally, the blank disk was put at a $10 \mathrm{~mm}$ distance of the meropenem disk and incubated overnight at $37^{\circ} \mathrm{C}$. The increased growth inhibition zone between the two disks was the indicator of MBL positive bacteria $(21,22)$. In addition, the AmpC disk test method was used to recognize betalactamase AmpC. Next, 0.5 McFarland suspension of $E$. coli ATCC 25922 on the Mueller-Hinton culture medium was passaged and a cefoxitin disk $(30 \mu \mathrm{g})$ was placed on the plate near a blank disk (Whatman paper) that was exposed to normal saline. Then, colonies resistant to meropenem were inoculated in the blank disk. A positive result for the production of AmpC $\beta$-lactamase was based on the observation of a dentin in the growth area of the cefoxitin disk in the vicinity of the blank disk (21-23).

\section{Determination of MBL genes}

The positive isolates for carbapenemase enzymes were used to investigate VIM and IMP genes by the polymerase chain reaction (PCR). Hence, their DNA concentrates were extracted by boiling. In the boiling process, $1 \mathrm{~mL}$ of sterile distilled water was poured in a $1.5 \mathrm{~mL}$ microtube. Then, 4-5 fresh bacterial colonies were dissolved and the microtube was placed on a heated plate at $95^{\circ} \mathrm{C}$ for 15 minutes to boil. Next, the samples were centrifuged at $14000 \mathrm{rpm}$ for 5 minutes and the transparent liquid containing bacterial DNA was removed for PCR. In this study, Acinetobacter baumannii AC54/97 and Pseudomonas aeruginosa PO510 were a positive control for IMP and VIM genes (24). Further, 16rrRNA was considered as internal control (25). 
The primers used for VIM and IMP genes were designed by Oligo 7 software (Table 1).

\section{Statistical analysis}

Descriptive statistics (i.e., frequency, percentage, mean, and standard deviation) and inferential statistics (Fisher's exact test) were analyzed and $P<0.05$ was considered as the significance level.

\section{Results}

In the disk diffusion method, 21 isolates of $E$. coli were resistant and 5 isolates were intermediate for meropenem and imipenem. Furthermore, 21 isolates of E. coli were resistant $(\mathrm{MIC} \geq 4 \mu \mathrm{g} / \mathrm{mL})$ to meropenem and imipenem antibiotics in the E-test.

\section{Modified Hodge test}

Resistant strains to meropenem and imipenem by E-test were used in MHT to detect carbapenemase enzymes and $15(18.75 \%)$ isolates of $E$. coli were positive $(P<0.001)$. Figure 1 shows the positive and negative samples by MHT.

\section{EDTA disk synergy test}

Escherichia coli isolates resistant to carbapenemase antibiotics were used in the EDS test to produce carbapenemase enzymes. In this test, $15(18.75 \%)$ isolates were positive $(P<0.001)$. Figure 2 illustrates two separate plates (1 \& 2) that are side by side and considered as negative and positive isolates for the EDS test, respectively.

\section{AmpC disk test}

The presence of a notch in the side of the disk reflects the production of AmpC $\beta$-lactamase by $E$. coli, which is on the blank disk (Figure 3, AmpC positive). In this test, 8 isolates $(10 \%)$ of $E$. coli were AmpC beta-lactamase $(P<0.001)$. Table 2 presents the results of phenotypic tests for detecting carbapenemase enzymes.

The results were similar for disk diffusion and E-Test. Moreover, among the tests for detecting carbapenemases enzymes, the MHT and EDS demonstrated the same

Table 1. Primers Used for VIM and IMP by PCR

\begin{tabular}{lc}
\hline Primer Sequence $\left(\mathbf{5}^{\prime}\right.$ to $\left.\mathbf{3}^{\prime}\right)$ & Size $(\mathbf{b p})$ \\
\hline VIM-1F TGGTTGTATACGTCCCGTCA & 206 \\
VIM-1 R TGTGTGCTGGAGCAAGTCTA & \\
IMP-1 F TAACGGGTGGGGCGTTGTTCCT & 179 \\
IMP-1 R CGCCCGTGCTGTCGCTATGAAA & \\
\hline
\end{tabular}

Note. VIM: Verona integron-encoded metallo- $\beta$-lactamase; IMP: imipenemase; PCR: polymerase chain reaction.

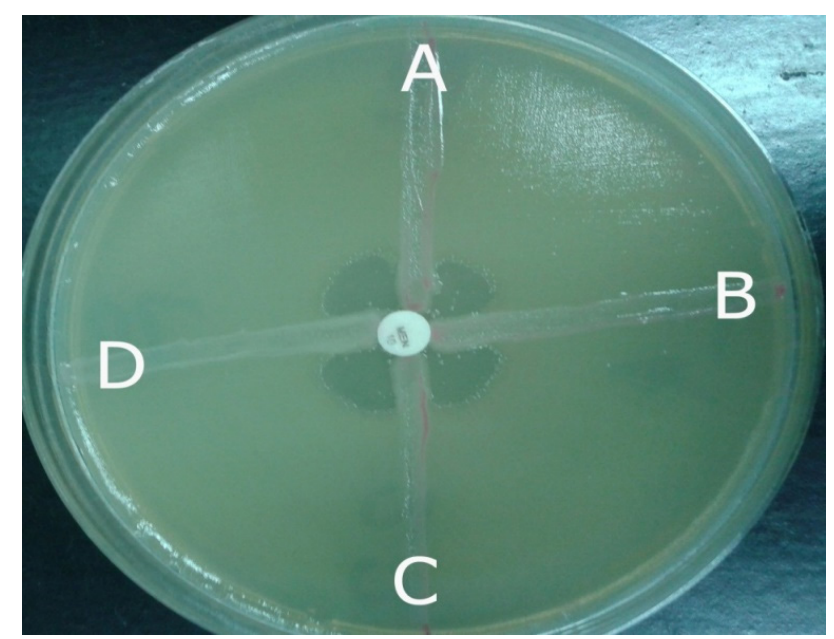

Figure 1. Modified Hodge test. E. coli ATCC25922 causes leaf clover halo in the inhibition zone around meropenem as a negative control by the Metallo-beta-lactamase enzyme production. $\mathrm{A}$ is the positive control strain of Klebsiella pneumonia (ATCC BAA-1705). In addition, $\mathrm{D}$ and $\mathrm{B}$ are positive $E$. coli strains and $\mathrm{C}$ is the negative isolate.

results, while their results differed from that of the AmpC test.

\section{Polymerase chain reaction}

The electrophoresis technique was performed on the $8 \%$ polyacrylamide gel after completing the PCR steps. After electrophoresis, the poly acryl amide gel was stained with the silver nitrate method (Figure 4). The results of this test are shown in Tables 3 and 4.

\section{Discussion}

Today, the resistance of bacteria to various antibiotics has become a global problem due to the uncontrolled administration of different antibiotics for treating bacterial infections. Hence, resistant strains are selected dramatically (26-28). Resistance to antibiotic therapy in patients with UTIs is considered as an example of increased antimicrobial resistance (12). Antimicrobial resistance has always been a major concern for human health (6), affecting patients throughout the world (29). MBL encoding genes are found more frequently on integrins, especially Class 1 integrin, which has the genetic mobility of these elements and promotes the release of these enzymes in different parts of a hospital (30). Similar to studies in Columbian, Japanese, and Italian hospitals, VIM and IMP were observed without the use of carbapenem antibiotics, which confirms the

Table 2. Results of phenotypic tests for the detection of MBL enzymes

\begin{tabular}{lccccc}
\hline Isolates $(\mathbf{N})$ & Disk-difiusion & E-Test & MHT & EDS Test & AmpC Disk Test \\
\hline 80 & $21(26.25 \%)$ & $21(26.25 \%)$ & $15(18.75 \%)$ & $15(18.75 \%)$ & $8(10 \%)$ \\
\hline Note. MBL: metallo-beta-lactamase; E-test: & Epsilon test; MHT: modified Hodge test; EDS: EDTA disk synergy test.
\end{tabular}



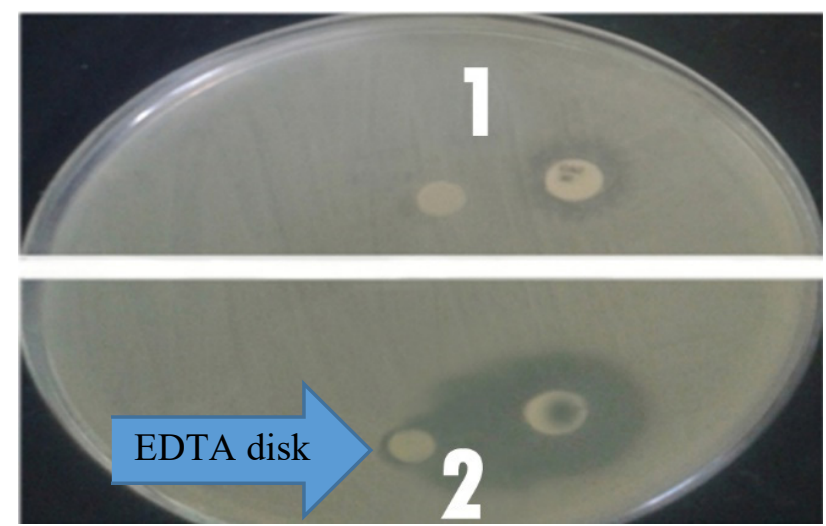

Figure 2. Ethylenediaminetetraacetic acid (EDTA) disk synergy test. At plate 2, increasing the diameter of the inhibition zone around the disk containing EDTA (blank) and between two discs of meropenem (right) and blank (left) represents Metallo-beta-lactamase production by bacteria in the presence of EDTA, inhibits the production of enzymes, and increases the diameter of the halo between the two disks.

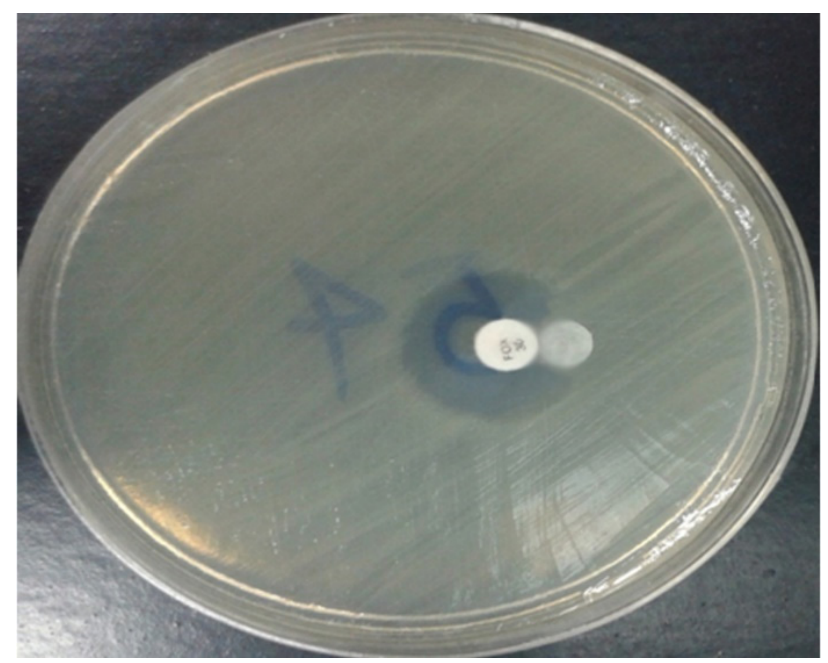

Figure 3. AmpC disk test. The notches on the side of the disk represent AmpC B-lactamase producing E. coli, which prevented the creation of a complete halation around the cefoxitin disk by E. coli ATCC 25922.

transfer of these genes between different species of bacteria $(31,32)$. In this study, the prevalence of resistance to meropenem and imipenem in E. coli strains was 26\%. In a study by Sahin et al, the production of MBL enzymes in 43 isolates of E. coli and Klebsiella (collected from various parts of the hospital) was evaluated during which 35 strains were carbapenem-resistant and produced MBL enzymes (33). The Center for Disease Control has supplied the MHT for MBL-producing Enterobacteriaceae isolates in order to detect MBL enzymes. Although this test has a high potential for detecting carbapenemase enzymes, the results are sometimes false positive by CTX-M and AmpC due to the partial hydrolysis of the carbapenemse (34). In

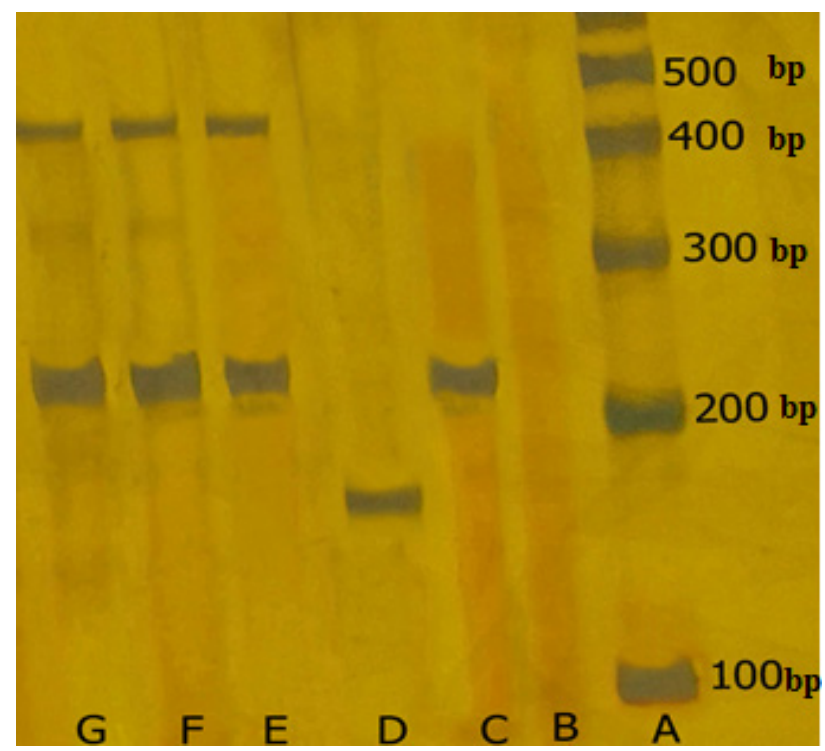

Figure 4. Colored gel of polyacrylamide. Column A: Ladder bp; B: Negative Control; C: Positive control of Verona integron-encoded Metallo- $\beta$-lactamase (VIM) gene (P. aeruginosa PO510); D: Positive control of imipenemase (IMP) gene (A. baumannii AC54 / 97); $\mathrm{E}$, $\mathrm{F}$, and $\mathrm{G}$ columns of VIM gene and internal control gene 16srRNA without IMP.

the MHT, the results are more reliable when used with the imipenem disk with 0.5 molar EDTA or $50 \mathrm{mM}$ ZNSO4 (35). The results of previous studies showed that MHT has less positive outcomes compared to the other tests, ranging from $14.8 \%$ to $51.16 \%$ (35-37), which is consistent with our results. In this study, 21 and 15 isolates of E. coli were positive by E-test and MHT method, respectively. In the research by Kumar et al, 28 isolates resistant of E. coli to meropenem (carbapenem) were reported and 6 isolates were positive by the MHT test, which indicates the low accuracy of MHT in detecting MBL strains (38). In addition, Sahin et al studied 43 strains of Klebsiella and E. coli and found that 35 strains were positive for the MHT, which were not reported in any of the VIM and IMP genes after the PCR (33). In this study, 15 isolates were positive in producing MBLs by the EDS test. Further, Kanchanadevi and Sekaran concluded that between 76 isolates of E. coli, 25 were resistant to imipenem using the E-test and 12 isolates were carbapenemase producing by the EDS test (39). AmpC is a cephalosporinase enzyme that leads to the hydrolysis of cefamycins (cefoxitin and cefotetan), which is a component of broad-spectrum beta-lactamase. To detect the AmpC beta-lactamase enzyme, AmpC disk testing can routinely be used in clinical laboratories. These laboratories have difficulty to appropriately detect this enzyme and there is little knowledge about the importance of this enzyme, which has led to the outbreak of these bacterial strains and even to treatment failure. In a study conducted in India, this enzyme was present in hospitalized strains, which 
Table 3. Abundance VIM and IMP Genes

\begin{tabular}{|c|c|c|c|c|c|c|}
\hline \multirow{2}{*}{$\begin{array}{c}\text { Isolates }(\mathbf{N}) \\
80\end{array}$} & \multicolumn{2}{|c|}{ VIM-1 } & $P$ value & \multicolumn{2}{|c|}{ IMP-1 } & $P$ value \\
\hline & $\begin{array}{c}\text { Positive } \\
19(23.75 \%)\end{array}$ & $\begin{array}{l}\text { Negative } \\
61(76.2 \%)\end{array}$ & $<0.001$ & $\begin{array}{c}\text { Positive } \\
0\end{array}$ & $\begin{array}{l}\text { Negative } \\
80(100 \%)\end{array}$ & $<0.001$ \\
\hline
\end{tabular}

Note. VIM-1: Verona integron-encoded metallo- $\beta$-lactamase 1 ; IMP-1: imipenemase 1.

Table 4. The relationship between resistance to meropenem and imipenem and the presence of VIM and IMP genes in E. coli

\begin{tabular}{lcc}
\hline \multirow{2}{*}{ Gene } & Meropenem and Imipenem Resistant $(\mathbf{N}=\mathbf{2 1})$ \\
\cline { 2 - 3 } & Positive & Negative \\
\hline VIM & 19 & 2 \\
IMP & 0 & 21 \\
Total & 19 & 23 \\
\hline
\end{tabular}

Note. VIM:Verona integron-encoded Metallo- $\beta$-lactamase; IMP: Imipenemase.

was more common in the Acinetobacter spp. Further, the prevalence was $6.97 \%$ for $E$. coli $(39,40)$. In our research, 8 isolates produced AmpC beta-lactamase among E. coli isolates, which is consistent with the results of the study of Koshesh et al in Kerman. In total, 2\% AmpC $\beta$-lactamase was positive among the 105 samples of $E$. coli isolated from UTI (41).

The presence of VIM and IMP genes with PCR was also evaluated in our study. The frequency of the VIM gene in E. coli isolates was $23.75 \%$ and the IMP gene was not found in E. coli strains. Based on the reports of Mosavian and Koraei, the frequency of the IMP gene in the strains of Klebsiella, E. coli, and Enterobacter was zero (42). In the study conducted by Tawfik et al, 15 strains of MBL-producing bacteria were reported by phenotypic methods. All the strains were VIM positive, but the IMP gene was not found in any of these strains. The results of the above-mentioned studies are in line with those of our study, showing a low prevalence of IMP genes in E. coli (43). Furthermore, Zeighami et al evaluated the molecular prevalence of VIM and IMP genes among 100 isolates and found that the prevalence was $41 \%$ and zero for Klebsiella and E. coli samples, respectively, indicating the different outbreaks of these genes in bacteria and regions (44). Similarly, Mushi et al investigated 227 bacterial samples including Klebsiella, E. coli, and Pseudomonas aeruginosa. After PCR, the highest frequency was attributed to IMP and VIM genes with 49 and 28 cases of IMP and VIM, respectively, which is also consistent with our study results representing a lower abundance of VIM and IMP genes in E. coli compared to the other bacteria (45).

\section{Conclusion}

Due to the failure of antibiotic treatment and increased therapeutic charges, especially in UTIs, all microbiology laboratories should now be able to identify MBLsproducing bacteria. In the absence of new antibiotics for treating infections caused by Gram-negative bacteria resistant to several antibiotics, the carbapenem will be ineffective in the near future. In addition, the treatment failure of these infections is predictable since these antibiotics are the preferred drug in treating multi-drug resistant bacteria. Resistance to carbapenem antibiotics is a growing problem and they might be replaced by novel antibiotics in the near future. Eventually, to control the emission of MBL-producing strains and the resistance of these microorganisms, it is necessary to come up with an appropriate treatment for these patients.

Conflict of Interests

None.

\section{Acknowledgments}

This study is a research project approved by Shahrekord University of Medical Sciences (approval No. 2057) on March 6, 2016. We, hereby, gratefully thank all the people who assisted us in conducting this work. In addition, the authors wish to thank Mr. H. Argasi at the Research Consultation Center of Shiraz University of Medical Sciences for his invaluable assistance in editing this manuscript.

\section{Ethical Statement}

The study protocol was obtained by the ethics code of IR.SKUMS. REC.1394.282 from Shahrekord University of Medical Sciences.

\section{Authors Contribution}

FK, AG and BZ: design of study; KN, AG, BZ, FK and MV: acquisition of data; $A G$ and MR: evaluation of data and preparation of the manuscript; AG, FK and MV assessment of data.

\section{Funding/Support}

This research was supported by the grant of the research project of the Shahrekord University of Medical Sciences (Shahrekord, Iran).

\section{References}

1. Yang J, Ye L, Wang W, Luo Y, Zhang Y, Han L. Diverse prevalence of $16 \mathrm{~S}$ rRNA methylase genes arm $A$ and $r m t B$ amongst clinical multidrug-resistant Escherichia coli and Klebsiella pneumoniae isolates. Int J Antimicrob Agents. 2011;38(4):348-51. doi: 10.1016/j.ijantimicag.2011.04.021.

2. Hodson CJ, Maling TM, McManamon PJ, Lewis MG. The pathogenesis of reflux nephropathy (chronic atrophic pyelonephritis). Br J Radiol. 1975; Suppl 13:1-26.

3. Gordon I, Barkovics M, Pindoria S, Cole TJ, Woolf AS. Primary vesicoureteric reflux as a predictor of renal damage in children hospitalized with urinary tract infection: a systematic review and meta-analysis. J Am Soc Nephrol. 2003;14(3):739-44. doi: 10.1097/01.asn.0000053416.93518.63.

4. Roussey-Kesler G, Gadjos V, Idres N, Horen B, Ichay L, Leclair $\mathrm{MD}$, et al. Antibiotic prophylaxis for the prevention of recurrent urinary tract infection in children with low grade vesicoureteral reflux: results from a prospective randomized study. J Urol. 2008;179(2):674-9. doi: 10.1016/j.juro.2007.09.090.

5. Karchmer TB, Giannetta ET, Muto CA, Strain BA, Farr BM. A randomized crossover study of silver-coated urinary catheters 
in hospitalized patients. Arch Intern Med. 2000;160(21):32948. doi: 10.1001/archinte.160.21.3294.

6. Baroud M, Dandache I, Araj GF, Wakim R, Kanj S, Kanafani $Z$, et al. Underlying mechanisms of carbapenem resistance in extended-spectrum beta-lactamase-producing Klebsiella pneumoniae and Escherichia coli isolates at a tertiary care centre in Lebanon: role of OXA-48 and NDM-1 carbapenemases. Int J Antimicrob Agents. 2013;41(1):75-9. doi: 10.1016/j. ijantimicag.2012.08.010.

7. Sahm DF, Thornsberry C, Mayfield DC, Jones ME, Karlowsky JA. Multidrug-resistant urinary tract isolates of Escherichia coli: prevalence and patient demographics in the United States in 2000. Antimicrob Agents Chemother. 2001;45(5):1402-6. doi: 10.1128/aac.45.5.1402-1406.2001.

8. Ko KS, Suh JY, Peck KR, Lee MY, Oh WS, Kwon KT, et al. In vitro activity of fosfomycin against ciprofloxacin-resistant or extended-spectrum beta-lactamase-producing Escherichia coli isolated from urine and blood. Diagn Microbiol Infect Dis. 2007;58(1):111-5. doi: 10.1016/j.diagmicrobio.2006.11.015.

9. Green VL, Verma A, Owens RJ, Phillips SE, Carr SB. Structure of New Delhi metallo-beta-lactamase 1 (NDM-1). Acta Crystallogr Sect F Struct Biol Cryst Commun. 2011;67(Pt 10):1160-4. doi: 10.1107/s1744309111029654.

10. Fernández $A$, Pereira MJ, Suárez JM, Poza $M$, Treviño $M$, Villalón $\mathrm{P}$, et al. Emergence in Spain of a multidrug-resistant Enterobacter cloacae clinical isolate producing SFO1 extended-spectrum beta-lactamase. J Clin Microbiol. 2011;49(3):822-8. doi: 10.1128/jcm.01872-10.

11. Meletis G, Tzampaz E, Protonotariou E, Sofianou D. Emergence of Klebsiella pneumoniae carrying bla(VIM) and bla(KPC) genes. Hippokratia. 2010;14(2):139-40.

12. Cagnacci S, Gualco L, Roveta S, Mannelli S, Borgianni L, Docquier JD, et al. Bloodstream infections caused by multidrug-resistant Klebsiella pneumoniae producing the carbapenem-hydrolysing VIM-1 metallo-beta-lactamase: first Italian outbreak. J Antimicrob Chemother. 2008;61(2):296300. doi: $10.1093 / \mathrm{jac} / \mathrm{dkm} 471$.

13. Zhang F, Zhu D, Xie L, Guo X, Ni Y, Sun J. Molecular epidemiology of carbapenemase-producing Escherichia coli and the prevalence of ST131 subclone H30 in Shanghai, China. Eur J Clin Microbiol Infect Dis. 2015;34(6):1263-9. doi: 10.1007/s10096-015-2356-3.

14. Goudarzi H, Taherpour A, Fallah F, Pourkaveh B, Erfanimanesh $\mathrm{S}$, Hashemi A. Laboratory detection of carbapenemases in gramnegative bacteria. Arch Clin Infect Dis. 2016;11(2):e32816. doi: 10.5812/archcid.32816.

15. Giske CG, Gezelius L, Samuelsen Ø, Warner M, Sundsfjord A, Woodford N. A sensitive and specific phenotypic assay for detection of metallo-beta-lactamases and KPC in Klebsiella pneumoniae with the use of meropenem disks supplemented with aminophenylboronic acid, dipicolinic acid and cloxacillin. Clin Microbiol Infect. 2011;17(4):552-6. doi: 10.1111/j.14690691.2010.03294.x.

16. Gholipour A, Soleimani N, Shokri D, Mobasherizadeh S, Kardi $M$, Baradaran A. Phenotypic and molecular characterization of extended-spectrum beta-lactamase produced by Escherichia coli, and Klebsiella pneumoniae isolates in an educational hospital. Jundishapur J Microbiol. 2014;7(10):e11758. doi: 10.5812/jjm.11758.

17. Thomson KS. Extended-spectrum-beta-lactamase, AmpC, and Carbapenemase issues. J Clin Microbiol. 2010;48(4):1019-25. doi: 10.1128/jcm.00219-10.

18. Khalil MAF, Elgaml A, El-Mowafy M. Emergence of multidrugresistant New Delhi metallo-beta-lactamase-1-producing Klebsiella pneumoniae in Egypt. Microb Drug Resist.
2017;23(4):480-7. doi: 10.1089/mdr.2016.0003.

19. Mlynarcik P, Roderova M, Kolar M. Primer evaluation for PCR and its application for detection of carbapenemases in Enterobacteriaceae. Jundishapur J Microbiol. 2016;9(1):e29314. doi: 10.5812/jjm.29314.

20. Adwan G, Bourinee H, Othman S. Prevalence of metallo-betalactamases producing Escherichia coli isolated from north of Palestine. J Microbiol Antimicrob Agents. 2016;2(1):9-15.

21. Amjad A, Mirza I, Abbasi S, Farwa U, Malik N, Zia F. Modified Hodge test: a simple and effective test for detection of carbapenemase production. Iran J Microbiol. 2011;3(4):18993.

22. Lee K, Chong Y, Shin HB, Kim YA, Yong D, Yum JH. Modified Hodge and EDTA-disk synergy tests to screen metallobeta-lactamase-producing strains of Pseudomonas and Acinetobacter species. Clin Microbiol Infect. 2001;7(2):88-91. doi: 10.1046/j.1469-0691.2001.00204.x.

23. Goel V, Hogade S, Karadesai S. Prevalence of extendedspectrum beta-lactamases, AmpC beta-lactamase, and metallobeta-lactamase producing Pseudomonas aeruginosa and Acinetobacter baumannii in an intensive care unit in a tertiary care hospital. Journal of the Scientific Society. 2013;40(1):2831. doi: 10.4103/0974-5009.109691.

24. Shahcheraghi F, Abbasalipour M, Feizabadi M, Ebrahimipour G, Akbari N. Isolation and genetic characterization of metallobeta-lactamase and carbapenamase producing strains of Acinetobacter baumannii from patients at Tehran hospitals. Iran J Microbiol. 2011;3(2):68-74.

25. Damavandi MS, Gholipour A, Latif Pour M. Prevalence of class D carbapenemases among extended-spectrum beta-lactamases producing Escherichia coli isolates from educational hospitals in Shahrekord. J Clin Diagn Res. 2016;10(5):DC01-5. doi: 10.7860/jcdr/2016/17722.7739.

26. Zowawi HM, Sartor AL, Balkhy HH, Walsh TR, Al Johani SM, AlJindan RY, et al. Molecular characterization of carbapenemase-producing Escherichia coli and Klebsiella pneumoniae in the countries of the Gulf cooperation council: dominance of OXA-48 and NDM producers. Antimicrob Agents Chemother. 2014;58(6):3085-90. doi: 10.1128/aac.02050-13.

27. Peleg $A Y$, Hooper DC. Hospital-acquired infections due to gram-negative bacteria. N Engl J Med. 2010;362(19):1804-13. doi: 10.1056/NEJMra0904124.

28. Podschun R, Ullmann U. Klebsiella spp. as nosocomial pathogens: epidemiology, taxonomy, typing methods, and pathogenicity factors. Clin Microbiol Rev. 1998;11(4):589-603.

29. Essack SY. Treatment options for extended-spectrum betalactamase-producers. FEMS Microbiol Lett. 2000;190(2):1814. doi: 10.1111/j.1574-6968.2000.tb09283.x.

30. Kil KS, Darouiche RO, Hull RA, Mansouri MD, Musher DM. Identification of a Klebsiella pneumoniae strain associated with nosocomial urinary tract infection. J Clin Microbiol. 1997;35(9):2370-4.

31. Khorsi K, Messai Y, Hamidi M, Ammari H, Bakour R. High prevalence of multidrug-resistance in Acinetobacter baumannii and dissemination of carbapenemase-encoding genes blaOXA23-like, blaOXA-24-like and blaNDM-1 in Algiers hospitals. Asian Pac J Trop Med. 2015;8(6):438-46. doi: 10.1016/j. apjtm.2015.05.011.

32. Yatsuyanagi J, Saito S, Harata S, Suzuki N, Ito Y, Amano K, et al. Class 1 integron containing metallo-beta-lactamase gene blaVIM-2 in Pseudomonas aeruginosa clinical strains isolated in Japan. Antimicrob Agents Chemother. 2004;48(2):626-8. doi: 10.1128/aac.48.2.626-628.2004.

33. Sahin K, Tekin A, Ozdas S, Akin D, Yapislar H, Dilek AR, et al. Evaluation of carbapenem resistance using phenotypic and 
genotypic techniques in Enterobacteriaceae isolates. Ann Clin Microbiol Antimicrob. 2015;14:44. doi: 10.1186/s12941-0150105-1.

34. Swain B, Otta S, Rout S. Resistance pattern of Klebsiella from urinary tract infections in a tertiary care hospital. Indian Pract. 2015;68(2):18-22.

35. Peleg AY, Seifert H, Paterson DL. Acinetobacter baumannii: emergence of a successful pathogen. Clin Microbiol Rev. 2008;21(3):538-82. doi: 10.1128/cmr.00058-07.

36. Lee K, Lim YS, Yong D, Yum JH, Chong Y. Evaluation

of the Hodge test and the imipenem-EDTA double-disk synergy

test for differentiating metallo-beta-lactamase-producing

isolates of Pseudomonas spp. and Acinetobacter spp. J Clin

Microbiol. 2003;41(10):4623-9. doi: 10.1128/jcm.41.10.4623-

4629.2003.

37. John S, Balagurunathan R. Metallo beta lactamase producing Pseudomonas aeruginosa and Acinetobacter baumannii. Indian J Med Microbiol. 2011;29(3):302-4. doi: 10.4103/02550857.83918 .

38. Kumar S, Mehra SK. Performance of modified Hodge test and combined disc test for detection of carbapenemases in clinical isolates of enterobacteriaceae. Int J Curr Microbiol Appl Sci. 2015;4(5):255-61.

39. Kanchanadevi P, Sekaran SC. Importance of EDTA in the detection of metallo beta lactamase from imipenem resistant gram-negative bacilli. Int J Curr Microbiol Appl Sci. 2016;5(11):702-6. doi: 10.20546/ijcmas.2016.511.081.
40. Singhal S, Mathur T, Khan S, Upadhyay DJ, Chugh S, Gaind $R$, et al. Evaluation of methods for AmpC beta-lactamase in gram negative clinical isolates from tertiary care hospitals. Indian J Med Microbiol. 2005;23(2):120-4. doi: 10.4103/02550857.16053.

41. Koshesh M, Mansouri S, Hashemizadeh Z, Kalantar-Neyestanaki D. Identification of extended-spectrum beta-lactamase genes and AmpC-beta-lactamase in clinical isolates of Escherichia coli recovered from patients with urinary tract infections in Kerman, Iran. Arch Pediatr Infect Dis. 2017;5(2):e37968. doi: 10.5812/pedinfect.37968.

42. Mosavian M, Koraei D. Molecular detection of IMP carbapenemase-producing gram-negative bacteria isolated from clinical specimens in Ahvaz, Iran. Jentashapir J Cell Mol Biol. 2016;7(6):e36394. doi: 10.17795/jjhr-36394.

43. Tawfik AF, Shibl AM, Aljohi MA, Altammami MA, AlAgamy $\mathrm{MH}$. Distribution of Ambler class A, B and D betalactamases among Pseudomonas aeruginosa isolates. Burns. 2012;38(6):855-60. doi: 10.1016/j.burns.2012.01.005.

44. Zeighami H, Haghi F, Hajiahmadi F. Molecular characterization of integrons in clinical isolates of betalactamase-producing Escherichia coli and Klebsiella pneumoniae in Iran. J Chemother. 2015;27(3):145-51. doi: 10.1179/1973947814y.0000000180.

45. Mushi MF, Mshana SE, Imirzalioglu C, Bwanga F. Carbapenemase genes among multidrug resistant gram negative clinical isolates from a tertiary hospital in Mwanza, Tanzania. Biomed Res Int. 2014;2014:303104. doi: 10.1155/2014/303104. 